\title{
Voltaire, Writings of 1758-1760
}

\section{Franco Piva}

\section{(2) OpenEdition}

\section{Journals}

\section{Electronic version}

URL: http://journals.openedition.org/studifrancesi/6306

DOI: 10.4000/studifrancesi.6306

ISSN: 2421-5856

\section{Publisher}

Rosenberg \& Sellier

\section{Printed version}

Date of publication: 1 November 2010

Number of pages: $557-558$

ISSN: 0039-2944

\section{Electronic reference}

Franco Piva, "Voltaire, Writings of 1758-1760", Studi Francesi [Online], 162 (LIV | III) | 2010, Online since 30 November 2015, connection on 09 January 2021. URL: http://journals.openedition.org/ studifrancesi/6306 ; DOI: https://doi.org/10.4000/studifrancesi.6306

This text was automatically generated on 9 January 2021.

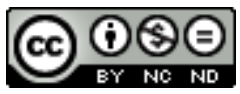

Studi Francesi è distribuita con Licenza Creative Commons Attribuzione - Non commerciale - Non opere derivate 4.0 Internazionale. 


\title{
Voltaire, Writings of 1758-1760
}

\author{
Franco Piva
}

\section{REFERENCES}

VOLTAIRE, Writings of 1758-1760, Oxford, Voltaire Foundation, 2009 («Les CEuvres complètes de Voltaire» 49B), pp. XXVII+465.

1 Le opere contenute in questo volume sono nate tutte, come succede peraltro spesso per Voltaire, da avvenimenti esterni, di fronte ai quali lo scrittore francese ha reagito da par suo. La più importante è certamente rappresentata dalla tragedia Tancrède, una pièce la cui composizione è strettamente correlata con la decisione degli attori del Théâtre Français di liberare finalmente il palco dalle numerose banquettes che l'ingombravano e che avevano progressivamente ristretto lo spazio riservato agli attori, fino al punto di trasformare la tragedia francese in una «tragédie de paroles», riducendo al massimo l'azione che non aveva, per l'appunto, abbastanza spazio per svilupparsi e manifestarsi. Ciò aveva impedito alla tragedia francese di soddisfare l'amore sempre più evidente degli spettatori per lo spettacolo, o per un'azione che fosse più fortemente caratterizzata. La liberazione della scena fu sentita da Voltaire, già da anni impegnato a tentare di trarre la tragedia francese dalle secche in cui l'avevano confinata le condizioni nelle quali essa veniva rappresentata (oltre che le modeste qualità di molti degli epigoni di Racine, il grande modello al quale la maggior parte di loro si erano ispirati), come un'importante possibilità di rinnovare la tragedia francese attraverso un'azione più robusta che permettesse, da un lato, di soddisfare il gusto del pubblico per lo spettacolo, dall'altro di «toucher son cœur», oltre che parlare al suo «esprit». L'argomento della nuova tragedia fu tratto da quel Medioevo che, per altro verso, consentiva di riallacciare i rapporti con quella storia nazionale, dalla quale, a dire il vero, Voltaire aveva già tratto azioni e personaggi in grado di suscitare l'interesse degli spettatori e che i recenti rovesci subiti, sul mare come sulla terraferma, dalle truppe francesi rendevano ancora più attuale. Per raggiungere meglio il suo scopo, Voltaire pensò addirittura di intervenire sulla scrittura stessa della pièce; adottando, se non la prosa invocata da qualcuno, i celebri «vers croisés» destinati a 
sostituire i monotoni alessandrini della tragedia più tradizionale. Lo spazio più ampio consentiva anche di portare sulla scena gruppi interi di comparse, e di dare maggiore importanza al décor. Ora, di fronte a queste nuove possibilità offerte a scrittori e ad attori, Voltaire ebbe, come fa ben notare John S. HENDERSON nella lunga introduzione che precede l'edizione critica, un atteggiamento ambiguo: attratto da tutte queste possibilità, lo scrittore si mostrò però anche cosciente dei rischi che esse comportavano; soprattutto e sopra tutte, quella di trasformare la tragedia in uno spettacolo da baraccone. Di qui le sue continue raccomandazioni agli attori, a Mlle Clairon in particolare, di non esagerare con la pantomime, come allora si diceva, e ai decoratori perché non abusassero degli ornements che, se dovevano suscitare l'interesse degli spettatori, non dovevano però mai distrarli dalle parole stesse della tragedia, in cui i versi, pur nella forma più libera in cui Voltaire li aveva disposti, avevano sempre il compito di conservare al dettato poetico quella dignità alla quale la tragedia non doveva mai rinunciare. Pur con questi limiti, Tancrède rappresentò tuttavia un momento fondamentale non soltanto nella produzione poetica di Voltaire, ma anche per l'intero teatro francese; come ha fatto notare J. S. Henderson in una pagina della sua dotta introduzione, nonostante i timori e le resistenze di Voltaire, «the prominence he gives to the pomp and splendour makes Tancrède a landmark of eighteenth-century dramatic production, which tended increasingly to stimulate visual pleasure».

2 Le altre opere contenute in questo volume trassero invece origine da un'altra circostanza. Come ricorda bene John MALLINSON nella «Preface» generale, gli anni ai quali fa riferimento questo volume furono anni molto difficili per i philosophes e per la battaglia che stavano portando avanti. La parte più tradizionale della società francese del tempo, sostenuta dai poteri costituiti, reagì infatti in maniera pesante alla presenza sempre più ingombrante e sempre più audace dei philosophes: da un lato, la condanna al rogo del De l'Esprit di Helvétius e la revoca del privilège dell'Encyclopédie, la potente macchina da guerra messa in piedi dai philosophes, dall'altro i duri attacchi di cui gli stessi furono oggetto da parte di Palissot e della potente Compagnia di Gesù. Tutto ciò rischiava di far naufragare il progetto di rinnovamento messo in atto dai philosophes, che anzi rischiavano di fare la fine che, nell'antichità greca, aveva fatto Socrate, al quale sempre più spesso essi vennero in quegli anni difficili assimilati. Da qui non solo il grande revival che conobbe la figura del filosofo greco, ma anche la decisione di Voltaire di portare quella mitica figura sulla scena. Ne nacque la Mort de Socrate, un testo di notevole interesse al quale il futuro Patriarca di Ferney non riuscì tuttavia mai, come bene spiega Raymond TROUSSON che ne ha curato l'edizione critica, a dare una forma soddisfacente, se non definitiva: per difficoltà tecniche certamente, ma anche per la scarsa propensione di Voltaire a prendere fino in fondo le difese di un gruppo di uomini, Diderot in testa, di cui condivideva grosso modo gli obiettivi ma ai quali rimproverava però anche eccessi dai quali egli avrebbe preso progressivamente, e strategicamente, le distanze.

Più diretta, e più forte, la reazione di Voltaire fu contro quella Compagnia di Gesù dalla quale pure egli aveva tanto ricevuto negli anni giovanili e con la quale aveva anche cercato, fino ad un certo punto, di mantenere buoni rapporti. Sempre più difficilmente tuttavia, specie dopo che a dirigere il giornale della Compagnia era stato chiamato il padre Berthier, che non nascose la sua insofferenza di fronte agli attacchi, peraltro sempre più frequenti e duri, che lo scrittore era venuto sferrando negli ultimi tempi alla religione. Fu lotta dura e senza quartiere, da una parte come dall'altra: agli attacchi 
sempre più espliciti dei Mémoires de Trévoux, Voltaire rispose con dei pamphlets contro il direttore stesso del giornale dei Gesuiti. Il presente volume ne presenta due: la Relation de la maladie, de la confession, de la mort et de l'apparition du jésuite Berthier e la Relation du voyage de frère Garassise, neveu de fère Garasse, successeur de frère Berthier; et ce qui s'ensuit, en attendant ce qui s'ensuivra; di cui David SMITH e Jean ORSONI hanno predisposto non solo, e con la solita cura, l'edizione critica ma anche un'attenta analisi stilistica. La verve satirica di Voltaire ha infatti modo di esplicarsi in questi scritti in tutta la sua tremenda capacità corrosiva; al punto che la Relation de la maladie, de la confession, de la mort et de l'apparition du jésuite Berthier è stata spesso additata come modello, ed esempio della scrittura satirica di Voltaire.

4 Completano il volume, predisposto con la cura alla quale gli editori delle «CEuvres complètes de Voltaire» ci hanno ormai abituati, alcuni scritti minori, e l'Indice delle opere citate. 